\title{
A handbook for modeling hippocampal circuits
}

\section{Michael E. Hasselmo*}

Center for Memory and Brain, Department of Psychology and Program in Neuroscience, Boston University, Boston, MA, USA

*Correspondence: hasselmo@bu.edu

\section{A book review on \\ Hippocampal microcircuits: a computational modeler's resource book}

by Vassilis Cutsuridis, Bruce Graham, Stuart Cobb and Imre Vida (eds.), Springer, New York, 2010, 450 pages. ISBN: 1441909958

In recent years, most areas of neuroscience research have moved beyond the scope of theories that can be described by text alone. Full understanding of the dynamics of neural circuits requires computational modeling to address the complex interaction of populations of excitatory and inhibitory neurons in guiding behavior. Yet the number of experimental researchers far exceeds the number of active modelers, and the time necessary to read the full experimental literature would easily consume the lifespan of the active modelers.

Hippocampal microcircuits: a computational modelers resource book serves to ease the process of mastering the literature within one of the most productive and active fields of neuroscience. This book facilitates the development of hippocampal models by distilling the essential features of research into an accessible and up-to-date format. The data summarized in this book covers the full scope of research on the hippocampus, starting with the anatomical connectivity, morphology, and intrinsic properties of hippocampal neurons, progressing through different neurotransmitter and neuromodulatory systems, and then focusing in detail on the computational modeling of hippocampal physiology.

No single researcher can be an expert in all areas, so the modeler must draw upon the expertise of individual researchers. However, review articles by experts commonly focus on the forefront of research without providing a full summary of the current state of knowledge. In contrast, the editors of this volume, Cutsuridis, Graham, Cobb, and Vida, have done an excellent job of ensuring that the chapters in this book are written in a clear, pedagogical manner that concisely summarizes the existing knowledge with clear subheadings.
As an overview of data for computational modeling, the book contains accessible and comprehensive reviews of experimental data on the hippocampus. The renowned hippocampal anatomist, Menno Witter, provides an overview of the anatomy of hippocampus and associated structures. In addition, the book contains excellent, well-structured review chapters on the morphology (Vida) and intrinsic physiology (Martina) of hippocampal neurons, as well as glutamatergic (Toth) and GABAergic transmission (Bartos et al.) and synaptic plasticity (Mellor). These chapters provide an outstanding resource to enhance the knowledge base of even highly experienced computational neuroscientists.

The modeling chapters provide a stepby-step approach to each subject, providing a cookbook for the modeler to program cellular and circuit simulations based on the knowledge of the experts. The editors have obtained outstanding expert assistance from specialists in all of the relevant domains of simulation. For example, several excellent chapters address the modeling of intrinsic properties of cells within individual hippocampal subregions. Poirazi provides an overview of the detailed modeling of hippocampal region CA1 neurons, Migliore describes modeling of hippocampal region CA3 neurons, Fransen describes modeling of entorhinal neurons, and Skinner and Saraga describe modeling of inhibitory interneurons. Accessible appendices at the end of each chapter provide the full equations and parameters for these models.

Likewise, the editors have recruited the experts on circuit level models to provide an overview of circuit level modeling. Of particular importance, they have focused on development of network simulations that utilize interactions of biophysically realis- tic compartmental simulations. Kopell and colleagues address biophysical modeling of gamma and theta rhythm oscillations. Graham, Cutsuridis, and Hunter address biophysical modeling of associative memory function in hippocampal areas CA1 and CA3. Models of associative memory function have strongly influenced experimental research on region $\mathrm{CA} 3$ and region CA1, particular with regard to their theoretical function in pattern completion. Morgan and Soltesz address modeling of the dentate gyrus and epileptiform activity. Again, the equations and parameters of the model are provided in the appendix of each chapter. This format will allow models to be constructed based on the information from this single book, saving the reader from extensive searching through a range of cited articles. Thus, the book can serve as a single, comprehensive reference for the full scope of modeling from cellular to circuit dynamics.

There has been a traditional gap between cellular level intrinsic models, and circuit models that address network function using synaptic modification. The editors help to bridge this gap by providing detailed chapters on biophysical modeling of synaptic modification mechanisms, including a chapter on models of the biophysical mechanisms underlying long-term potentiation and a chapter by Gerkin, Bi, and Rubin on phenomenological modeling of spike-timing-dependent plasticity (STDP).

Given the breadth of this field, there are bound to be some topics that cannot be addressed in detail. In particular, the description could have included more details about the dynamical properties of neurons, though this has already been covered extensively in other books. The 
description of entorhinal cortex could benefit from more detailed discussion of the relationship of intrinsic cellular properties to grid cell firing properties, extending the brief discussion by Fransen. The book includes useful chapters by Eichenbaum and Erdi addressing the functional role of grid cells and place cells, but the potential link between the unit firing properties of neurons and their intrinsic properties are not addressed.

The book provides a very practical overview that includes addressing the currently available simulation environments that range from more general neural modeling packages such as NEURON, GENESIS, and XPP to packages focused on hippocampal modeling such as Surf-Hippo. The chapter also addresses the ever growing importance of neural databases such as ModelDB/ NeuroDB at Yale and the NeuroMorpho. org web site developed by Ascoli at George Mason University.

This book will prove highly valuable as a resource both for expert modelers as well as for new researcher constructing their first model of hippocampal cells and circuits. Hippocampal Microcircuits will advance the field by allowing functional hypotheses to be implemented in computational simulations that incorporate the full scope of available data on a particular mechanistic question.

Received: 09 December 2010; accepted: 04 January 2011; published online: 20 January 2011.

Citation: Hasselmo ME (2011) A handbook for modeling hippocampal circuits. Front. Comput. Neurosci. 5:2. doi: 10.3389/fncom.2011.00002

Copyright $\odot 2011$ Hasselmo. This is an open-access article subject to an exclusive license agreement between the authors and Frontiers Media SA, which permits unrestricted use, distribution, and reproduction in any medium, provided the original authors and source are credited. 